\title{
The Effects of Low HER 2/neu Expression on the Clinicopathological Characteristics of Triple-Negative Breast Cancer Patients
}

\author{
Mehdi Dehghani ${ }^{1 *}$, Pedram Keshavarz ${ }^{2}$, Abdolrasoul Talei ${ }^{3}$, Majid Akrami ${ }^{3}$, \\ Sedighe Tahmasebi ${ }^{3}$, Akbar Safaie ${ }^{4}$, Maryam Ghanbari ${ }^{5}$
}

\begin{abstract}
Background: Triple-negative breast cancer (TNBC) is an aggressive type of breast cancer (BC), and its diagnosis is associated with negative expression of hormone receptors and HER2/neu. It consists of 10-20\% of all BCs diagnosed. Methods and materials: This study focuses on three groups with different pathology: group one showed complete triple-negative HER2 expression with IHC of BC; groups two and three included patients with ER-, PR-, and HER2 ${ }^{1+}$, and ER-, PR-, and HER $2^{2+}$ with a negative FISH test. These three groups were compared from the point of prognosis, which consisted of tumor size, patients' age, lymphatic, vascular and perineural invasion, organ metastasis, number of lymph nodes involvement, and the survival rate. Results: A total of 459 TNBC patients were enrolled, of which 268 were placed in the HER $2^{0}$ group, 146 in the HER $2^{1+}$ group, and 45 in the HER2 ${ }^{2+}$ group. Distant metastasis and recurrence rate were more common in HER $2^{\circ}$ patients, but bone metastasis was more common in patients with low HER2 expression. All patients with HER $2^{0}$ had a smaller tumor size at the time of $\mathrm{BC}$ diagnosis in comparison to patients in the low HER2 expression group. Patients with HER $2^{2+}$ had less lymphatic and vascular invasion as well as axillary lymph nodes involvement, but larger tumor size at presentation, resulting in a lower rate of recurrence and higher overall survival. Conclusion: The findings revealed that patients with HER $2^{2+}$ had better outcome in comparison to the patients with HER2 $2^{0}$ and HER $2^{1+}$. Furthermore, the results showed that many patients with HER $2^{2+}$ expression were not basal-like and had good prognosis amongst TNBC patients.
\end{abstract}

Keywords: Triple-negative breast cancer- hormone receptor- HER2/neu- basal-like tumor

Asian Pac J Cancer Prev, 21 (10), 3027-3032

\section{Introduction}

Breast cancer $(\mathrm{BC})$ is a heterogeneous malignancy amongst females in the USA (Zeichner et al., 2016), and the second cause of cancer death worldwide (first is lung cancer) (Gradishar et al., 2016; DeSantis et al., 2016) Today, there are about 1.5 million $B C$ patients, and 200,000 BC patients are diagnosed every year (Ghislain et al., 2016) While chemotherapy, radiotherapy, and other drug hormonal therapies have led to improvement of BC treatment, almost $25 \%$ of patients with primary detectable $\mathrm{BC}$ still have recurrence with or without metastasis to other organs, such as bone, liver, lung, etc (Noman et al., 2016).

Triple-negative breast cancer (TNBC) is a type of BC, which has higher mortality due to its more local recurrence and distant metastasis (Asano et al., 2017), which becomes more resistance to chemotherapy than other types of $\mathrm{BC}$, resulting in shorter survival (Wang et al., 2015; Li et al., 2017) TNBC diagnosis was made possible by the negative expression of hormone receptors and human epidermal growth factor receptor 2 (HER2) neu, which was reported to account for $10-20 \%$ of all BC cases (Denkert et al., 2017) TNBC is categorized by the absence of three main biomarkers, i.e. estrogen and progesterone receptors, low expression of HER 2 by immunohistochemistry (IHC), and lack of amplification by fluorescence in situ hybridization (FISH) test. The absence of these features means that the hormonal agents and anti-HER2 treatment methods cannot be successful in treating TNBC (Kim et al., 2018; Collignon et al., 2016). The epidermal growth factor receptor (EGFR) activation stimulates cell proliferation and provide protection against apoptosis by activating protein kinase pathways (Li et al., 2017; Kim et al., 2017)

Several methods, such as IHC, chromogenic in situ

${ }^{1}$ Hematology Research Center, Department of Hematology and Medical oncology, Shiraz University of Medical Sciences, Shiraz, Iran. ${ }^{2}$ Department of Radiology, Medical Imaging Research Center, Shiraz University of Medical Sciences, Shiraz, Iran. ${ }^{3}$ Breast Diseases Research Center, Shiraz University of Medical Sciences, Shiraz, Iran. ${ }^{4}$ Department of Pathology, School of Medicine, Shiraz University of Medical Sciences, Shiraz, Iran. ${ }^{5}$ Department of Internal Medicine, Shiraz University of Medical Sciences, Shiraz, Iran.*For Correspondence: dehghanim@sums.ac.ir 
hybridization (CISH) and FISH are now available to quantify HER2/neu expression.(Goud et al., 2012) Hence, the aim of this study was to compare three groups of TNBCs, namely complete negative HER 2 expression by IHC, minimal HER $2^{(1+)}$ expression, and moderate HER $2^{(2+)}$ expression by IHC and the negative FISH test for HER2 amplification. The HER 2 negative group included HER $2^{\circ}$, HER $2^{1+}$, and HER $2^{2+}$, based on differences in the staining intensity and the negative FISH test in the HER $2^{2+}$. This study investigated the possible differentiations in the natural characteristics of TNBC with different low HER2 expressions.

\section{Materials and Methods}

In this retrospective cross-sectional study, a total of 459 cases of triple-negative breast carcinoma were identified from Jun 2002 to Jun 2018 in Breast Disease Clinic, affiliated with Shiraz University of Medical Sciences (SUMS). The clinical and histopathological evaluation of the patients was done by examining a number of pathological features, including age, tumor size, axillary lymph node involvement, and vascular, perineural and lymphatic invasions. Other pathological characteristics, including tumors' grade, and type of tumor histology, were also evaluated. Distant metastasis was determined and confirmed by history and imaging, including ultrasonography, computerized tomography (CT) scan, and whole-body bone scan. PET-CT scan was used to determine a special case while fine-needle aspiration or needle biopsy was used to confirm the suspicious and difficult cases. Estrogen receptor (ER) and progesterone receptor (PR) status were also noted by IHC.

We selected only patients with negative estrogen and progesterone hormone, but we divided them into three distinct groups according to their HER2 statuses. Based on IHC, but with negative FISH test for HER2 amplification, the HER 2 was determined to be completely negative in the first group, minimal (1+) in the second group, and moderate $(2+)$ positive in the third group. The inclusion criteria were patients with negative estrogen and progesterone hormone, patients with HER $2^{0}$, HER $2^{1+}$, or HER $2^{2+}$ expression, and a negative HER 2 amplification by FISH. The exclusion criteria were patients with positive hormone receptors and HER $2^{3+}$ by IHC or HER $2^{2+}$ and a positive amplification by the FISH test.

\section{Statistical analysis}

All statistical analysis was performed, using descriptive statistics and independent t-test. Pearson's Chi-square test was used to calculate the association between the variables. $\mathrm{P}$ values $<0.05$ were considered to be statistically significant. All statistical analyses were conducted, using SPSS version 21.0.

\section{Ethics statements}

In this study, all procedures involving human participation were performed in accordance with the ethical standards of the institutional and/or national research committee and the 1964 Helsinki Declaration and its later amendments or comparable ethical standards.
The study was approved by the Ethics Committee of Shiraz University of Medical Sciences, Shiraz, Iran (code: IR.SUMS.MED.REC. 1394.s40).

\section{Results}

In total, 459 TNBC patients joined the study, of which 268 were completely negative for the HER 2 expression, 146 patients were HER $2^{1+}$, and 45 patients were HER2 ${ }^{2+}$ and a negative HER 2 amplification by the FISH test. All patients were followed up from June 2002 through Jun 2018 , with a minimum of 16 days to a maximum of 15 years of surveillance and a median of $4.41 \pm 2.52$ years. The youngest patient, aged 24 was in the HER2 ${ }^{\circ}$ group while the oldest one aged 81 was in the HER $2^{1+}$ group. The mean age characteristics of the three distinct groups are presented in Table 1.

The evaluation of the histopathological characteristics of the patients and a comparison of the lymphatic invasion showed a minimal higher rate of invasion in the HER2 ${ }^{0}$ and HER $2^{1+}$ groups, but the difference was not statistically significant. $(\mathrm{P}=0.6)$ A comparison of the extent to which the right- and left-side breast were affected by the tumor showed that the right-side breast was affected more $66.6 \%$ $(\mathrm{n}=30)$ by the tumor in the HER $2^{2+}$, but the difference was not statistically significant $(\mathrm{P}=0.1)$. Therefore, it can be stated that in all three groups, both sides of the breast were equally affected by tumor. The type of surgery was affected by the patient's preference, size of the tumor, and the tumor stage. A comparison between groups revealed that patients in the HER2 ${ }^{1+}$ group had undergone more breast conservation surgery; however, the difference was not statistically significant $(P=0.7)$ (Table 1$)$.

The stage of disease at presentation was affected by the tumor size, but the lymph nodes involvement was the most significant factor in $\mathrm{BC}$ prognosis. The patients were also divided into three groups based on their tumor size, i.e. patients with tumor size less than $2 \mathrm{~cm}$ were allocated in the first group, patients with tumor sizes of equal or more than $2 \mathrm{~cm}$, and equal or less than $5 \mathrm{~cm}$ in the second group, and patients with tumor size more than $5 \mathrm{~cm}$ in the third group. The results indicated that in the HER $2^{\circ}$ group, $26.5 \%(\mathrm{n}=71)$ of patients belonged to the first group (tumor size $<2 \mathrm{~cm}), 58.6 \%(\mathrm{n}=157)$ to the second group (tumor size between 2 and $5 \mathrm{~cm}$ ), and $14.9 \%(\mathrm{n}=40)$ to the third group (tumor size $>5 \mathrm{~cm}$ ). It appeared that the overall size of tumor in the HER $2^{\circ}$ group was smaller at $\mathrm{BC}$ presentation. On the other hand, tumor sizes of more than $5 \mathrm{~cm}$ were slightly more common in the HER $2^{1+}$ and HER $2^{2+}$ groups, with $17.8 \%(\mathrm{n}=22)$ and $22.2 \%(\mathrm{n}=10)$ of patients identified to have tumor sizes more than $5 \mathrm{~cm}$ in these two groups, respectively. (Table 1) Invasive ductal carcinoma (IDC) was the most predominant subtype of $\mathrm{BC}$ in all three types of cancer. About $69 \%(\mathrm{n}=185)$ of IDC was found in HER $2^{\circ}$ and a total of $72.3 \%(n=332)$ in all the groups together while the medullary carcinoma was prominent in the HER $2^{\circ}$ subtype with about $16.7 \%$ $(n=45)$ of cases, and $13.9 \%(n=64)$ of the total patients in all the groups. The lobular and other pathologic subtypes are not common in TNBC.

After tumor size, the most important factor in the 
Table 1. The Clinical Characteristics of the Patients and Tumor Samples Used in the Study

\begin{tabular}{|c|c|c|c|c|c|}
\hline Characteristics & $\mathrm{N}$ & $\mathrm{HER}^{0} \mathrm{~N}(\%)$ & HER2 $2^{1+} \mathrm{N}(\%)$ & $\mathrm{HER}^{2+} \mathrm{N}(\%)$ & $\mathrm{P}$ \\
\hline \multicolumn{6}{|l|}{ Age } \\
\hline Mean age \pm St. deviation & & $46.5 \pm 12.14$ & $46.3 \pm 8.09$ & $48.6 \pm 6.83$ & N.S \\
\hline \multicolumn{6}{|l|}{ Tumor size (1)* } \\
\hline $\mathrm{T}<2 \mathrm{~cm}$ & 134 & $71(26.5)$ & $47(32.2)$ & $16(35.6)$ & N.S \\
\hline $2 \mathrm{~cm} \leq \mathrm{T} \leq 5 \mathrm{~cm}$ & 249 & $157(58.6)$ & $73(50)$ & $19(42.2)$ & \\
\hline $\mathrm{T}>5 \mathrm{~cm}$ & 76 & $40(14.9)$ & $26(17.8)$ & $10(22.2)$ & \\
\hline \multicolumn{6}{|l|}{ Lymph nodes invasion $(1)^{* *}$} \\
\hline $\mathrm{N}<3$ & 83 & $67(25)$ & $2(1.3)$ & $14(31.1)$ & N.S \\
\hline $3 \leq \mathrm{N} \leq 10$ & 32 & $22(8.2)$ & $9(6.1)$ & $1(2.2)$ & \\
\hline $\mathrm{N}>10$ & 4 & $3(3)$ & $1(0.7)$ & 0 & \\
\hline without lymph node involvement & 340 & $176(65.6)$ & $134(91.7)$ & $30(66.6)$ & \\
\hline \multicolumn{6}{|l|}{ Side of invasion $* * *$} \\
\hline Right & 234 & $135(50.3)$ & $69(47.2)$ & $30(66.6)$ & N.S \\
\hline Left & 225 & $133(49.6)$ & $77(52.7)$ & $15(33.3)$ & \\
\hline \multicolumn{6}{|l|}{ Surgery $* * * *$} \\
\hline $\mathrm{BCS}$ & 267 & $156(58.9)$ & $87(59.6)$ & $24(53.3)$ & N.S \\
\hline MRM & 192 & $112(41.8)$ & $59(40.4)$ & $21(46.6)$ & \\
\hline Total & 459 & 268 & 146 & 45 & N.S \\
\hline
\end{tabular}

Table 2. The Rate of Involvement of the Lymph Nodes in the Three Separate Groups

\begin{tabular}{lccccc}
\hline Cancer type & Mean age & $\begin{array}{c}\text { Lymph nodes } \\
\text { involvement less than 3 }\end{array}$ & $\begin{array}{c}\text { Lymph nodes } \\
\text { involvement (3-10) }\end{array}$ & $\begin{array}{c}\text { Lymph nodes } \\
\text { involvement more than 10 }\end{array}$ & $\begin{array}{c}\text { Survival (Alive) } \\
\text { in 4.4 years }\end{array}$ \\
\hline HER2 $^{2}$ & 46.5 & $77.80 \%$ & $23.90 \%$ & $3.30 \%$ & $86.10 \%$ \\
HER2 $^{1+}$ & 46.3 & $71.40 \%$ & $25.70 \%$ & $2.90 \%$ & $86.30 \%$ \\
HER2 $^{2+}$ & 48.6 & $93.30 \%$ & $6.70 \%$ & 0 & $95.50 \%$ \\
\hline
\end{tabular}

staging of BC was lymph nodes involvement. The patients were also categorized according to the numbers of lymph

Table 3. The Histopathological Characteristics of the Patients who Took Part in the Study

\begin{tabular}{lccccc}
\hline Characteristics & $\mathrm{N}$ & $\begin{array}{c}\mathrm{HER}^{0} \\
\mathrm{~N}(\%)\end{array}$ & $\begin{array}{c}\text { HER2 } \\
\mathrm{N}(\%)\end{array}$ & $\begin{array}{c}\text { HER2 } \\
\mathrm{N}(\%)\end{array}$ & $\mathrm{P}$ \\
\hline Histopathology & & & & & \\
$\quad$ Insitu & 9 & $4(1.5)$ & $3(2.1)$ & $2(4.4)$ & N.S \\
IDC & 332 & $185(69)$ & $114(78)$ & $33(73.3)$ & N.S \\
ILC & 4 & $3(1.1)$ & $1(0.7)$ & 0 & N.S \\
Mucinous & 3 & $2(0.7)$ & $1(0.7)$ & 0 & N.S \\
Medullary & 64 & $45(16.7)$ & $12(8.2)$ & $7(15.5)$ & N.S \\
Papillary & 3 & $3(1.1)$ & 0 & 0 & N.S \\
Others & 44 & $26(9.7)$ & $15(10.2)$ & $3(6.6)$ & N.S \\
Total & 459 & 268 & 146 & 45 & N.S \\
Local recurrence $*$ & & & & \\
Yes & 82 & $53(21.1)$ & $24(17.3)$ & $5(11.4)$ & N.S \\
No & 352 & $198(79.8)$ & $115(82.7)$ & $39(88.6)$ & \\
Metastasis ** & & & & & \\
Yes & 56 & $40(14.9)$ & $11(7.5)$ & $5(11.1)$ & N.S \\
No & 403 & $228(85.1)$ & $135(92.4)$ & $40(88.8)$ & \\
\hline
\end{tabular}

IDC, Invasive ductal carcinoma; ILC, Invasive lobular carcinoma; N.S, Not significant; * P value, $0.2 ; * * \mathrm{P}$ value, 0.6 . nodes involvement either by sentinel lymph node biopsy or axillary lymph nodes dissection. The first group consisted of patients with less than three lymph nodes involvement, the second group patients with equal and more than three, and equal and less than ten lymph nodes involvement, and the third group patients with more than ten lymph nodes involvement. The most lymph nodes involvement was observed in the HER2 ${ }^{\circ}$ group with 92 patients and $3(3 \%)$ of the HER $2^{0}$ patients had more than 10 lymph nodes involvement, which was the worst prognosis and an ominous factor for local recurrence and distant metastasis. In the HER $2^{2+}$ group, we observed that most of the patients had none or less than three lymph nodes involvement. In comparing the involvement of the axillary lymph nodes, especially in patients with more than ten lymph nodes, which is associated with a shorter lifespan and we observed that the lowest rate is in the HER $2^{2+}$ group and also the lowest mortality rate is seen in this group, although it is not much different in terms of statistics (Table 2).

With regard to the distant metastasis, 56 out of 459 patients had distant metastasis and the most common sites were lung, liver, and bone, respectively. The distant metastasis rate was slightly more common in the HER2 ${ }^{0}$ group $14.9 \%(\mathrm{n}=40)$ than in the HER2 ${ }^{2+} 11.1 \%(\mathrm{n}=5)$ and HER $2^{1+} 7.5 \%(\mathrm{n}=11)$ groups. Also, brain metastasis was slightly more common in the HER $2^{0}$ group, but the 
Table 4. Tumor Invasion and Metastasis to Various Organs

\begin{tabular}{|c|c|c|c|c|c|c|c|c|c|c|}
\hline & $\begin{array}{l}\text { Lymphatic } \\
\text { invasion }\end{array}$ & $\begin{array}{l}\text { Vascular } \\
\text { invasion }\end{array}$ & $\begin{array}{l}\text { Perineural } \\
\text { invasion }\end{array}$ & $\begin{array}{c}\text { Tumor size } \\
\text { more than } 5 \mathrm{~cm}\end{array}$ & $\begin{array}{c}\text { Liver } \\
\text { metastasis }\end{array}$ & $\begin{array}{c}\text { Lung } \\
\text { metastasis }\end{array}$ & $\begin{array}{c}\text { Bone } \\
\text { metastasis }\end{array}$ & $\begin{array}{c}\text { Brain } \\
\text { metastasis }\end{array}$ & $\begin{array}{l}\text { Multiple sites } \\
\text { of metastasis }\end{array}$ & $\begin{array}{l}\text { Total } \\
\text { case }\end{array}$ \\
\hline $\mathrm{HER}^{0}{ }^{0}$ & $14.20 \%$ & $2.50 \%$ & $5 \%$ & $14.90 \%$ & $3.40 \%$ & $4.70 \%$ & $2.60 \%$ & 1.15 & $3.10 \%$ & 268 \\
\hline HER $2^{1+}$ & $16.50 \%$ & $2.40 \%$ & $3.40 \%$ & $17.80 \%$ & $1.40 \%$ & 0 & $1.40 \%$ & $0.70 \%$ & $3.50 \%$ & 146 \\
\hline HER $2^{2+}$ & $9.80 \%$ & 0 & 9.85 & $22.20 \%$ & 0 & $6.70 \%$ & $2.20 \%$ & 0 & 0 & 45 \\
\hline
\end{tabular}

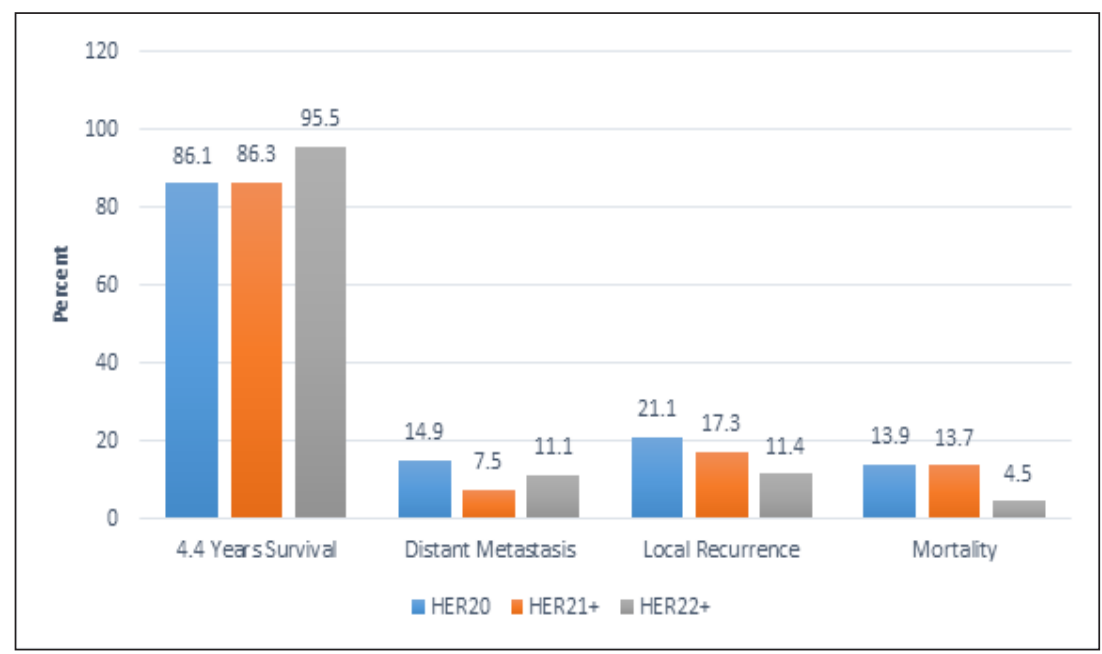

Figure 1. Comparing Survival Rate, Distant Metastasis, Local Recurrence and Mortality Rate

differences were not significant. The following results were obtained based on the local recurrence: $21.1 \%(\mathrm{n}$ $=53)$ of patients in the HER20, $17.3 \%(\mathrm{n}=24)$ in the HER $2^{1+}$ group, and $11.4 \%(n=5)$ in the HER2 ${ }^{2+}$ group had local recurrence. This meant that the local recurrence rate was slightly higher in the HER $2^{0}$ patients compared to HER $2^{1+}$ and HER2 ${ }^{2+}$ groups (Table 3 ).

In the study of tumor invasion, we observed the lowest rate of vascular and lymphatic invasion, as well as the lowest rate of metastasis to the liver and brain, and multiple metastases to various organs are seen in the HER2 $^{2+}$ group (Table 4).

Selecting radiotherapy as a therapeutic modality is multifactorial, including tumor size, axillary lymph nodes involvement and the type of surgery. In this study, no significant difference was observed between the three study groups with respect to radiation. Majority of the patients, $84.2 \%, 85 \%$, and $83.8 \%$, in the HER2 ${ }^{\circ}$ HER2 $^{1+}$, and HER $2^{2+}$ groups, respectively, received radiation. From June 2002 to June $2018,86.1 \%(n=231)$ of the HER2 ${ }^{0}$ patients were alive and the mortality rate in this group was $13.8 \%(\mathrm{n}=37)$; in the HER $2^{1+}$ group, $86.3 \%(\mathrm{n}=126)$ were alive during the same period and the mortality rate was $13.6 \%(n=20)$ for this group. In the HER $2^{2+}$ group, only $4.4 \%(n=2)$ of patients were died and $95.5 \%(n=$ 43) were still alive. As the above findings indicate, the mortality rate was very low in the HER $2^{2+}$ group, but the difference between the groups was not statistically significant $(\mathrm{P}=0.2)$ (Figure 1$)$

\section{Discussion}

Breast cancer (BC) is referred to as type of mixed tumors with different actions, prognosis, and difficult to treat (Noman et al., 2016) Many studies reported that about $15 \%$ of BC patients are TNBC positive. This type of cancer is more aggressive than other $\mathrm{BC}$ types and has poorer prognosis compared to other BC types (Speers et al., 2017; Kucukzeybek et al., 2018) Recent studies revealed that $\mathrm{BC}$ heterogeneity extends to the classic IHC-based subtypes of ER, PR, and HER2 receptors. (Gradishar et al., 2016; Kurozumi et al., 2016) Nowadays, new treatment methods are suggested for TNBC according to new biomarkers and gene expression profile (Burstein et al., 2015; Sarin et al., 2016).

Our results suggest that the three groups in the present study were close to each other with regard to the investigated variables. For some parameters, such as metastasis, recurrence, and tumor size, the HER2 status did not significantly change in the BC prognosis. Regarding the local recurrence, the results showed a $21.1 \%$ for the HER $2^{0}$ group and $17.3 \%$ for the HER2 ${ }^{1+}$ group vs. $11.4 \%$ for the HER22+ group. With regard to the metastasis to solid organs, the findings showed $24.3 \%$ metastasis in the HER $2^{0}$ group vs. $8.9 \%$ in the HER2 ${ }^{1+}$ group and $7.5 \%$ in the HER2 ${ }^{2+}$ group. More solid organ metastasis was observed in the HER $2^{0}$ group, but was not significantly different between groups. A comparison of the three groups regarding the tumor size and axillary lymph nodes involvement indicated that there was a larger tumor size at presentation in the $\mathrm{HER}^{2+}$ patients, but more axillary lymph nodes involvement was observed in the HER $2^{0}$ group. These findings show more aggressive behavior in HER $2^{0}$ than in HER2 ${ }^{2+}$ patients.

A second comparison was made to gain more specific data about the HER2 status role in the prognosis of BC patients. The results indicated that $\mathrm{HER} 2^{2+}$ patients had a higher survival rate, a lower rate of lymph nodes 
involvement, less lymphatic invasion, and a lower rate of local recurrence. Both the current study and the study by Plasilova et al. revealed that HER2 ${ }^{2+}$ was a protective factor in $\mathrm{BC}$ patients. In other words, many $\mathrm{HER} 2^{2+}$ patients were not affected by the basal-like tumor.(Plasilova et al. 2016) Regarding the lymph node involvement, the findings revealed that the HER2 ${ }^{2+}$ status in BC meant that more lymph nodes were involved. It was also found that HER $2^{2+}$ patients had more perineural invasion, but less lymphatic invasion. All three groups had the same distribution regarding the three categories of tumor size. To be more specific, there were more patients in all the three groups who were classified in the category of $2-5 \mathrm{~cm}$, but the number was higher for the patients in the HER2 ${ }^{2+}$ group. Contrary to the expectation, TNBC group had a smaller tumor size (14.9\% in the HER20 group, $17.8 \%$ in the HER $2^{1+}$ group, and $22.2 \%$ in the HER $2^{2+}$ group had a tumor size more than $5 \mathrm{~cm}$ ). Other studies have shown that even small tumors seem to have a higher recurrence rate in the HER $2^{0}$ group, suggesting a correlation between larger tumor sizes and the increase of HER2 receptor status, and also between larger tumors and the HER2 ${ }^{2+}$ group.(Amar et al. 2010; Kaplan, Malmgren, and Atwood 2009) However, metastatic BC was more common in TNBC, and there was no significant difference between the HER $2^{1+}$ and HER $2^{2+}$ groups regarding metastatic BC. In another study, Schmidt et al. had divided TNBCs into three subtypes, i.e. HER2/neu score 0 , score 1, and score 2. The results of their study revealed that TNBC patients with a HER2/neu score of 0 had a significantly poorer outcome regarding disease-free survival.(Schmidt et al., 2016) Therefore, the identification of $\mathrm{HER} 2^{2+}$ in TNBC patients can play a significant role in the prognosis of this disease, which is a contribution of this study.

According to the tumor staging system and the evaluation of the tumor size, lymph nodes involvement, and distant metastasis, it became clear that patients with HER20 had more basal-like behavior and a more aggressive type of tumor; whereas patients with $\mathrm{HER}^{2+}$ had a less aggressive tumor and mostly were not basal-like tumor. Equally important, the results of this study showed that both the rate of recurrence and metastasis rate were higher in the TNBC patients (just bone metastasis was higher in the HER $2^{1+}$ or HER2 ${ }^{2+}$ with negative FISH); however, the TNBC patients generally had a smaller tumor size.

Several biomarkers, including cytokeratin 5/6, EGFR, and negative staining for both ER and HER2, which are highly sensitive to basal-like BC might account for these differences between the basal-like and triple-negative tumors. Additional biomarkers, such as cytokeratin 14 or 17, p53 stabilization, and higher mitotic indices, are associated with worse survival in triple-negative tumors. One potential strategy for confirming the basal-like tumors is the evaluation of the 50-gene expression profile to determine the molecular subtype; however, this methodology is not practical in many centers. It should be noted that many new molecular targets, including phenotypic and genomic analysis, are prognostic and might have therapeutic effects on TNBC (Johnstone et al., 2020; Shan et al., 2019) Irrespective of the new molecular and genomic markers that have significant effect on the prognosis of $\mathrm{BC}$, a more practical strategy for determining the basal-like TNBC and its prognosis is the use of HER2 by IHC to identify the protein expression surrogates for the basal-like gene signature (Toft and Cryns, 2011)

In conclusion, to conclude, it can be stated that HER2 ${ }^{2+}$ status makes the TNBC cells are less aggressive and result in a better prognosis. More research should be conducted to further our understanding the TNBC characteristics and its prognosis so that we can develop better treatment methods and easier ways to increase the survival rate of patients with this type of BC.

\section{Acknowledgments}

This article was extracted from Mrs. Maryam Ghanbari's thesis in partial fulfillment of the requirement for the degree of doctor of medicine (Proposal No. 94-01-01-9101). The authors would like to thank the staff of the Department of Breast Diseases Research Center, Shiraz University of Medical Sciences, Shiraz, for their kind cooperation in gathering data for this study. The authors wish to thank Mr. H. Argasi at the Research Consultation Center (RCC) of Shiraz University of Medical Sciences for his invaluable assistance in editing this manuscript.

\section{Conflicts of Interest Statement}

The authors certify that they have no affiliations with or involvement in any organization or entity with any financial interest, or non-financial interest, in the subject matter or materials discussed in this manuscript.

\section{References}

Amar S, Ann E, Mc C, et al (2010). Prognosis and outcome of small $(\leq 1 \mathrm{~cm})$, node-negative breast cancer on the basis of hormonal and HER-2 status. Oncologist, 15, 1043.

Asano Y, Shinichiro K, Wataru G, et al (2017). Expression and clinical significance of androgen receptor in triple-negative breast cancer. Cancer, 9, 4 .

Burstein MD, Anna T, Graham MP, et al (2015). Comprehensive genomic analysis identifies novel subtypes and targets of triple-negative breast cancer. Clin Cancer Res, 21, 1688-98.

Collignon, Laurence L, Helene S, Guy J ( 2016). Triple-negative breast cancer: treatment challenges and solutions. Breast Cancer Targets Ther, 8, 93.

Denkert C, Cornelia L, Andrew T, Gunter von M (2017). Molecular alterations in triple-negative breast cancer-the road to new treatment strategies. Lancet, 389, 2430-42.

DeSantis R, Carol E, Stacey AF, et al (2016). Breast cancer statistics, 2015: Convergence of incidence rates between black and white women. CA Cancer J Clin, 66, 31-42.

Ghislain I, Efstathios Z, Coens C, et al (2016). Health-related quality of life in locally advanced and metastatic breast cancer: methodological and clinical issues in randomised controlled trials. Lancet Oncol, 17, 294-304.

Goud KI, Seetha D, Vijayalaxmi K, et al (2012). Evaluation of HER-2/neu status in breast cancer specimens using immunohistochemistry (IHC) \& fluorescence in-situ hybridization (FISH) assay. Indian J Med Res, 135, 312.

Gradishar WJ, Benjamin OA, Balassanian R, et al (2016). Invasive breast cancer version $1.2016, \mathrm{NCCN}$ clinical practice guidelines in oncology. J Natl Compr Canc Netw, Asian Pacific Journal of Cancer Prevention, Vol $21 \mathbf{3 0 3 1}$ 
14, 324-54.

Johnstone CN, Pattison AD, Harrison PF, et al (2020). FGF13 promotes metastasis of triple-negative breast cancer. Int $J$ Cancer, 147, 230-43.

Kaplan HG, Judith AM, Mary A (2009). T1N0 triple negative breast cancer: risk of recurrence and adjuvant chemotherapy. Breast $J$, 15, 454-60.

Kim A, Min HJ, Soo JL, Young KB (2017). Mutations of the epidermal growth factor receptor gene in triple-negative breast cancer. J Breast Cancer, 20, 150-9.

Kim C, Ruli G, Emi S, et al (2018). Chemoresistance evolution in triple-negative breast cancer delineated by single-cell sequencing. Cell, 173, 879-93. e13.

Kucukzeybek A, Betul, Ibrahım VB, et al (2018). Prognostic significance of androgen receptor expression in HER2positive and triple-negative breast cancer. Polish J Pathol, 69, 157-68.

Kurozumi S, Padilla M, Kurosumi M, et al (2016). HER2 intratumoral heterogeneity analyses by concurrent HER2 gene and protein assessment for the prognosis of HER2 negative invasive breast cancer patients. Breast Cancer Res Treat, 158, 99-111.

Li L, Yahua Z, Hongyan Z, et al (2017). Association between oral contraceptive use as a risk factor and triple-negative breast cancer: A systematic review and meta-analysis. $\mathrm{Mol}$ Clin Oncol, 7, 76-80.

Noman AS, Uddin U, Rahman MZ, et al (2016). Overexpression of sonic hedgehog in the triple negative breast cancer: clinicopathological characteristics of high burden breast cancer patients from Bangladesh. Sci Rep, 6, 18830.

Plasilova ML, Hayse B, Killelea BK, et al (2016). Features of triple-negative breast cancer: Analysis of 38,813 cases from the national cancer database. Medicine, 95, e4614.

Sarin R, Khandrika L, Hanitha RNM, et al (2016). Epidemiological and survival analysis of triple-negative breast cancer cases in a retrospective multicenter study. Indian J Cancer, 53, 353.

Schmidt G, Gerlinger C, Juhasz-Böss I, et al (2016). Her2-neu score as a prognostic factor for outcome in patients with triple-negative breast cancer. J Cancer Res Clin Oncol, 142, 1369-76.

Shan J, Aziz C, et al (2019). Genetic variation in CCL5 signaling genes and triple negative breast cancer: Susceptibility and Prognosis Implications. Front Oncol, 9, 1328.

Speers F, Corey W, Benjamin C, et al (2017). Radiosensitization of androgen receptor (AR)-positive triple-negative breast cancer (TNBC) cells using seviteronel (SEVI), a selective CYP17 lyase and AR inhibitor. Am Soc Clinl Oncol, 35, e12102.

Toft G, Daniel J, Vincent LC (2011). Minireview: Basal-like breast cancer: from molecular profiles to targeted therapies. Mol Endocrinol, 25, 199-211.

Wang D, Yubao H, Tinghu Z, et al (2015). CDK7-dependent transcriptional addiction in triple-negative breast cancer. Cell, 163, 174-86.

Zeichner SB, Hiromi T, Keerthi G (2016). A review of systemic treatment in metastatic triple-negative breast cancer. Breast Cancer Basic Clin Res, 10, BCBCR. S32783.

This work is licensed under a Creative Commons AttributionNon Commercial 4.0 International License. 